\title{
Unexplained Practice Variation in Primary Care Providers' Concern for Pediatric Obstructive Sleep Apnea
}

\author{
Sarah Honaker ${ }^{\mathrm{a}}, \mathrm{PhD}$, Tamara Dugan ${ }^{\mathrm{b}}, \mathrm{MS}$, Ameet Daftary ${ }^{\mathrm{c}}$, MD MS, Stephanie Davis ${ }^{\mathrm{d}}$, MD \\ Chandan Saha ${ }^{\mathrm{e}}$, PhD, Fitsum Baye ${ }^{\mathrm{f}}$, MS, Emily Freeman ${ }^{\mathrm{g}}$, \& Stephen Downs ${ }^{\mathrm{h}}$, MD, MS
}

\begin{abstract}
Affiliations:
${ }^{a}$ Pulmonology, Allergy, and Sleep Medicine Department of Pediatrics Indiana University School of Medicine 705 Riley Hospital Drive, ROC 4270 Indianapolis IN 46202, USA smhonake@iupui.edu

${ }^{\mathrm{b}}$ Children's Health Services Research Department of Pediatrics Indiana University School of Medicine $410 \mathrm{~W} 10^{\text {th }}$ Street, HS Suite 2000

Indianapolis, IN 46202, USA

tmdugan@iu.edu

${ }^{\mathrm{c}}$ Department of Pediatrics

Pulmonology, Allergy, and Sleep Medicine 705 Riley Hospital Drive, ROC 4270

Indianapolis IN 46202, USA

adaftary@iupui.edu

${ }^{\mathrm{d}}$ Department of Pediatrics

Pulmonology, Allergy, and Sleep Medicine

705 Riley Hospital Drive, ROC 4270

Indianapolis IN 46202, USA

Davisstd386@iu.edu

${ }^{\mathrm{e}}$ Department of Biostatistics

Richard M. Fairbanks School of Public Health

Indiana University School of Medicine

$410 \mathrm{~W} 10^{\text {th }}$ Street, HS Suite 3000

Indianapolis, IN 46202, USA

cksaha@iu.edu

${ }^{\mathrm{f}}$ Department of Biostatistics

Richard M. Fairbanks School of Public Health

Indiana University School of Medicine

$410 \mathrm{~W} 10^{\text {th }}$ Street, HS Suite 3000
\end{abstract}

This is the author's manuscript of the article published in final edited form as:

Honaker, S., Dugan, T., Daftary, A., Davis, S., Saha, C., Baye, F., ... Downs, S. (2018). Unexplained Practice Variation in Primary Care Providers' Concern for Pediatric Obstructive Sleep Apnea. Academic Pediatrics. 
Indianapolis, IN 46202, USA

fbaye@iu.edu

${ }^{g}$ Indiana University Bloomington

107 S. Indiana Avenue

Bloomington, IN 47405, USA

emilfree@umail.iu.edu

Please note that Ms. Freeman is an undergraduate, thus no degree is listed.

${ }^{\text {h }}$ Children's Health Services Research

Department of Pediatrics

Indiana University School of Medicine

$410 \mathrm{~W} 10^{\text {th }}$ Street, HS Suite 2000

Indiana polis, IN 46202, USA

stmdowns@iu.edu

Address correspondence to: Sarah Honaker, Pulmonary, Allergy, and Sleep Medicine, Indiana, 705 Riley Hospital Drive, ROC 4270, Indianapolis, Indiana, smhonake@ iupui.edu, 317-9441345 .

Key Words: Computer Decision Support; Primary Care Provider; Sleep Disorders; Obstructive Sleep Apnea; Pediatric

Running Title: Pediatric Obstructive Sleep Apnea in Primary Care

Conflict of Interest: Stephen Downs is the co-inventor of CHICA and the president of Digital Health Solutions, LLC, a company created to license the CHICA software. Tamara Dugan is the chief technology officer of Digital Health Solutions. At present, there are not patents and no license agreements. None of the other authors have any conflicts of interest to disclose. 


\begin{abstract}
Objective: To examine primary care provider (PCP) screening practice for obstructive sleep apnea (OSA) and predictive factors for screening habits. A secondary objective was to describe the polysomnography (PSG) completion proportion and outcome. We hypothesized that both provider and child health factors would predict PCP suspicion of OSA.

Methods: A computer decision support system that automated screening for snoring was implemented in five urban primary care clinics in Indianapolis, Indiana. We studied 1086 snoring children between 1 and 11 years seen by 26 PCPs. We used logistic regression to examine the association between PCP suspicion of OSA and child demographics, child health characteristics, provider characteristics, and clinic site.
\end{abstract}

Results: PCPs suspected OSA in $20 \%$ of snoring children. Factors predicting PCP concern for OSA included clinic site $(\mathrm{p}<.01 ; \mathrm{OR}=0.13)$, Spanish language $(\mathrm{p}<.01 ; \mathrm{OR}=0.53)$, provider training $(\mathrm{p}=.01 ; \mathrm{OR}=10.19)$, number of training years $(\mathrm{p}=.01 ; \mathrm{OR}=4.26)$ and child age $(\mathrm{p}<.01)$, with the youngest children least likely to elicit PCP concern for OSA $(\mathrm{OR}=0.20)$. No patient health factors (e.g., obesity) were significantly predictive. Proportions of OSA suspicion were variable between clinic sites (range $6 \%$ to $28 \%$ ) and between specific providers (range $0 \%$ to $63 \%$ ). Of children referred for PSG ( $n=100), 61 \%$ completed the study. Of these, $67 \%$ had OSA. Conclusions: Results suggest unexplained small area practice variation in PCP concern for OSA amongst snoring children. It is likely that many children at-risk for OSA remain unidentified. An important next step is to evaluate interventions to support PCPs in evidence-based OSA identification. 


\section{What's New}

This study describes a computer decision support system that automates screening for snoring in primary care clinics, and examines factors that predicted clinical suspicion of obstructive sleep apnea (OSA). Findings suggest low proportions of provider suspicion of OSA and referral as well as unexplained practice variation. 
Obstructive Sleep Apnea (OSA) presents frequently in children and has been associated with sequelae that negatively impact the lives of affected youth. ${ }^{1-4}$ American Academy of Pediatrics (AAP) clinical practice guidelines include two recommendations particularly pertinent to the primary care setting: 1) screen all children for habitual snoring and perform a focused evaluation for those that are snoring; and 2) refer children with snoring and one or more additional signs or symptoms of OSA. ${ }^{5}$ However, studies consistently suggest low proportions of screening, diagnosis, and management of OSA in pediatric primary care. ${ }^{6}$ Most children are not regularly screened for snoring, with proportions ranging from $8 \%$ to $24 \% .^{7,8}$ When snoring is identified, proportions of management are low, and patient adherence to recommended referrals is undocumented. ${ }^{7,8}$ Low rates of screening and management for OSA are suggestive of unwarranted practice variation, in which variation cannot be explained on the basis of illness, medical evidence, or patient preference. ${ }^{9}$

Computer Decision Support Systems (CDSS) can be used to automate screening and provide clinical decision-making support to PCPs. One such CDSS, Child Health Improvement through Computer Automation (CHICA), has demonstrated improved PCP adherence to guidelines for screening and management in many areas of child health, including asthma ${ }^{10}$ and developmental delay. ${ }^{11}$ We introduced a novel module into the existing CHICA system (CHICA Snore) which automates screening for snoring.

The aims of this study are to 1) describe the proportion of snoring children for whom PCPs suspect OSA, within the context of an automated screening system; 2) examine predictive provider- and child-level factors; and 3) describe the proportion of children referred for PSG who 
complete the procedure within one year. We hypothesized that PCPs would be more likely to suspect OSA in children with known OSA risk factors (e.g., overweight). We further hypothesized that providers who were completed training more recently would be more likely to identify OSA.

Methods

\section{CHICA System}

CHICA, an innovative CDSS that has been operating in primary care clinics at our institution since 2004, is described elsewhere in detail. ${ }^{12-14}$ Briefly, parents receive a prescreening form (PSF) in the waiting room via electronic tablet at both sick and well child visits. The PSF consists of twenty health risk items tailored to the child based on age and prior medical history. With this information, CHICA generates up to six prompts for the PCP on a physician worksheet (PWS), which appears in the electronic health record (EHR). The physician can mark check boxes to document his or her response to the prompt, which is then imported into the child's EHR.

\section{CHICA Snore Module}

In September 2015, the CHICA Snore module was incorporated into the existing CHICA system. Caregivers received the following item: "Does [CHILD NAME] consistently snore three or more nights per week?" The frequency of three nights per week was selected based on AAP guidelines. ${ }^{5}$ The item was presented in English or Spanish, depending on the family's preferred language. An affirmative response automatically triggered a PCP prompt noting that the child snores and recommending evaluation for OSA. PCPs could endorse one of three responses in the prompt: child does not snore; I am concerned about OSA; I do not suspect OSA. 


\section{Setting and Participants}

This study was conducted between September 2015 and March 2016. Children ages 1-11.9 years presenting for a sick or well child visit were eligible. We included PCPs who responded ten or more times to the EHR prompt identifying a snoring child. Because the analysis included the impact of provider variables (e.g., years since training) on PCP decision-making, we estimated that at least ten responses were necessary to provide a sample adequate to estimate a PCP's typical response pattern. The CHICA Snore module was incorporated into five clinic sites in the Eskenazi Health System in Indianapolis, Indiana. Twenty-two attending physicians and 4 nurse practitioners participated in the project. The Institutional Review Board of the Indiana University School of Medicine approved this study and waived informed consent, as the screening for snoring was conducted as part of routine clinical practice.

\section{Data Collection and Analysis}

We extracted data from the CHICA system for eligible children whose caregivers were presented with the snoring screening item during the study time frame. Data were analyzed using SAS software, version 9.4 (SAS Institute, Inc., Cary, NC, USA). We calculated the caregiver response for the snoring item, the frequency at which caregivers endorsed snoring, PCP response to the associated prompt, and the frequency of specific PCP responses. Proportions of PCP suspicion of OSA by clinic site and by provider were also explored graphically.

The primary outcome was PCP response to the prompt (concern for OSA vs. do not suspect OSA). We also examined whether or not the PCP responded at all (any response vs. no response). Chi-square tests were used to explore bivariate associations between the outcomes and 
clinic site, child, and provider characteristics. The following child characteristics were analyzed: age group, gender, race/ethnicity (black, white, Hispanic, other/unknown), preferred caregiver language (English, Spanish), insurance status (Medicaid, other), BMI status (BMI percentile $<85$ or $\geq 85$ ), and presence or absence of ADHD symptoms. The presence of ADHD symptoms was defined as prior initiation of ADHD evaluation documented in CHICA. Because the outcome of the ADHD evaluation was often missing, ADHD diagnosis could not be used as a variable. Provider characteristics included gender, years since training (above and below median), and type of training (pediatrician, medicine/pediatrics physician, family medicine physician, nurse practitioner). Years since training was dichotomized as the data were skewed and had large variability. The clinic site variable consisted of the site at which the child was seen.

Because only a small proportion of providers (8\%) practiced at more than one clinic site, multiple children are nested under a single provider. Therefore, logistic regression with a random effects model was used to explore variables that were associated with PCP concern for OSA. Clinic site, child and provider characteristics were included in the model as fixed effects. Provider is included in the model as a random effect to account for correlation of outcome measures from the same provider.

Logistic regression modeling was used to examine the association between clinic site, child, and provider characteristics with the two outcomes. We developed three models based on the analyses of univariate associations with the outcomes, including variables with a p-value $<.25$. Model one includes clinic and patient characteristics. Model two includes clinic and provider characteristics. Model three includes clinic, patient, and provider characteristics with p-value < 
.25 at multivariate analyses (models one and two), and only the significant predictors and the clustering factor (clinic) were retained in the final multivariate model.

For 175 children for whom the PCP indicated concern for OSA, we reviewed the child's medical record at their primary care clinic, and also cross-checked the patient names with a list of all patient referred for a PSG from the start of the study (September 2015) until twelve months after the end of the study (March 2017). For children referred for a study, we identified the referral status (completed; currently scheduled; initiated but not completed; scheduled but not completed; never scheduled). For completed studies we reviewed the PSG report to extract the apnea hypopnea index (AHI), a measure commonly used to document OSA presence or absence as well as severity. These data were collected and managed using REDCap electronic data capture tools hosted at Indiana University School of Medicine.

\section{Results}

\section{Patient Flow}

Patient flow is summarized in Figure 1. Across the five clinic sites, caregivers of 8256 eligible children were presented with a snoring item. A total of 8135 caregivers responded, for an overall response of $99 \%$. Of those who responded, 2320 (29\%) of caregivers reported that their children snored three or more days per week.

During the study timeframe, no prompt was generated for seven snoring children because other health issues were prioritized for those children via the CHICA prioritization scheme. ${ }^{14}$ Thirtynine PCPs or residents received a total of 2313 automated prompts in response to a positive screen for snoring. Thirteen providers, primarily residents, were excluded because they received 
fewer than ten prompts over the data collection period, resulting in the exclusion of 30 children in subsequent analyses and leaving 26 attending physicians and nurse practitioner providers for the final analysis. Fifteen children were excluded because the PCP checked multiple boxes, making results difficult to interpret. After these exclusions, 2268 children remained. Of these, PCPs responded to prompts for 1086 children, a $48 \%$ response.

\section{Child Characteristics}

Participating children with PCP responses for snoring $(n=1086)$ were $49 \%$ female and between the ages of 1 and 11.9 years with a mean age of $5.5(\mathrm{SD}=3.3)$ years. Child race and ethnicity were reported as follows: 44\% Black, 5\% White, 28\% Hispanic and 21\% Other. Spanish was the preferred language for $40 \%$ of families, with $93 \%$ of these children being seen by a bilingual PCP for the visit at which the screening occurred. Most children (84\%) had Medicaid insurance, and $36 \%$ were overweight or obese (Table 1 ).

\section{Provider Characteristics}

A total of 26 primary care providers were included in the analysis. Providers were $31 \%$ female with a mean of 15.4 years (Range 3-42) since completion of residency training. Nurse practitioners comprised $15 \%(n=4)$. Physicians were trained in pediatrics $(n=15)$, pediatrics and internal medicine $(n=3)$ or family medicine $(n=4)$.

\section{PCP Response to Prompt}

PCP responses to the checkboxes on the physician worksheet for the 1086 children were as follows: $20 \%$ concern for OSA; 59\% OSA not suspected; and 21\% snoring not reported. 
Proportions of concern for OSA varied among the five clinic sites (range $6 \%$ to $28 \%$ ). The variability amongst individual providers was even greater (0\% to $63 \%$; Figure 2 ).

\section{Predictors of PCP Concern for OSA}

The univariate association analyses with PCP concern for OSA showed five significant predictors: clinic, child's age, language, provider training and number of training years, of which all remained significant in the final model. In the final logistic regression model (Table 2) factors predicting PCP concern for OSA included clinic site $(\mathrm{p}<.01)$, language $(\mathrm{p}<.01)$, provider training $(\mathrm{p}=.013)$, number of training years $(\mathrm{p}=.011)$ and child's age $(\mathrm{p}<.001)$. For child age, the oldest children (ages 8.3-11.9 years) were most likely to elicit PCP concern for OSA compared to the youngest (ages 1.0-2.5 years; $\mathrm{OR}=0.2$ ) and middle group of children (ages 2.65.2 years; $\mathrm{OR}=0.5$ ). Children of caregivers who identified Spanish as the preferred language were less likely to elicit concern for OSA compared to children of caregivers who identified English as the preferred language $(\mathrm{OR}=0.53)$. Providers with higher than median number of years since training (14 years) had 4 times higher odds of identifying OSA problem compared with providers below the median for years since training $(\mathrm{OR}=4.3)$. Providers trained in both internal medicine and pediatrics were more than ten times more likely to suspect OSA compared to those trained in pediatrics $(\mathrm{OR}=10.19)$. No patient health factors (e.g., obesity status or ADHD symptoms) were significantly predictive. Among snoring children for whom the PCP responded to the prompt, $37 \%$ were overweight or obese $(n=396)$ and $18 \%(n=190)$ had ADHD symptoms, resulting in $44 \%(n=478)$ of children who met one or both criteria.

\section{Predictors of PCP Response}


In unadjusted analyses, younger child age, not having ADHD, Hispanic and other race/ethnicity (compared to non-Hispanic white) were associated with higher odds of PCP response to the prompt. In the final logistic regression model, only younger child age $(\mathrm{OR}=1.58)$ and race/ethnicity $(\mathrm{OR}=1.81$ for Hispanic children and 1.89 for other $)$ remained significant.

\section{Referral Outcome}

Of snoring children for whom PCPs noted a concern for OSA $(n=175), 46 \%$ were referred for PSG, $12 \%$ were referred to ENT, $18 \%$ received a different management plan (i.e., starting or increasing nasal spray, allergy medication, montelukast, or similar), and 24\% did not receive any type of management plan or referral. Of those referred, only $61 \%$ completed the PSG (despite scheduling availability), 17\% were scheduled but cancelled or missed the appointment, $16 \%$ were contacted for a PSG but never scheduled, $2 \%$ initiated but did not complete the PSG, and 4\% had plans for a PSG referral documented in the chart but no evidence of any referral, scheduled appointment, or completed study. OSA was identified in $51 \%$ of children completing the PSG, with a range of severity: $56 \%$ mild $(2.0<\mathrm{AHI}>4.99) ; 12 \%$ moderate $(5<\mathrm{AHI}>9.99) \%$; and $32 \%$ severe $(\mathrm{AHI}>10)$.

\section{Discussion}

This study demonstrates the feasibility of using an automated system to screen patients for snoring in busy primary care clinics. Caregiver response to the screening item was high (99\%), resulting in the identification of more than two-thousand snoring children. PCPs subsequently noted that approximately $21 \%$ of snoring children in fact did not snore, which may have been related to parental misinterpretation of the screening question or parental responding based on 
the child's current status (i.e., child with an acute respiratory illness) instead of the child's usual health status. PCP response to the automated prompt was modest (48\%), though similar to the provider response in other studies using the CHICA system. ${ }^{15,16}$ Further, the proportion of children who were evaluated by their provider for snoring with CHICA (48\%, as defined by PCP response to the prompt) is much higher than in previous studies in primary care systems that did not use an automated screening tool. ${ }^{6}$ Previous research on the CHICA system has found that providers are less likely to respond to prompts on topics in which they have less comfort and experience, ${ }^{15}$ and OSA is an area in which both pediatricians ${ }^{17,18}$ and nurse practitioners ${ }^{19}$ receive little training.

Despite automated identification of snoring children, this study found a low proportion of PCP concern for OSA. There was a large degree of practice variation in PCP concern for OSA: some PCPs never endorsed concern for OSA whereas others endorsed concern for more than half of patients. While there are some systematic differences in the children seen by individual providers (e.g., bilingual providers are more likely to see Latino children) as well as systematic demographic differences between clinic sites, it is highly unlikely that this would account for the degree of observed differences in provider practice, particularly given that the proportion of suspicion is amongst already identified snoring children. Further, clinical variables that might be expected to predict which children would elicit concern for OSA (e.g., obesity, ADHD symptoms) were not in fact significant predictors. Altogether, these findings strongly support unwarranted practice variation in PCP decision-making around OSA. Practice variation is generally considered a strong indicator of lack of consensus and suboptimal quality in clinical 
practice. $^{20}$ Encouragingly, when PCPs did suspect OSA, 76\% of the time there was a documented management plan in the child's medical record, most frequently a referral for PSG.

Also concerning is that only $61 \%$ of children referred for PSG had completed the study within a year. As the PSG wait time during the study time period was never longer than 3 months, availability of appointments was likely not a factor. Additional research would be helpful in identifying modifiable factors that contribute to poor completion.

Age and caregiver language were the only significant child demographic predictors. The oldest children (ages 8.3 to 11.9 years) were most likely to elicit concern for OSA, though the literature does not support a higher prevalence in this age group. ${ }^{21}$ The finding that children whose caregivers preferred Spanish language were half as likely to elicit concern for OSA is consistent with previous work suggesting that non-English speakers in the United States may receive a lower quality of care. ${ }^{22}$ While Spanish-speaking families in our clinics are often seen by one of several bilingual providers this is not always the case, increasing the likelihood of communication difficulties. Alternately, there may be cultural difference in perception of snoring as concerning. Contrary to our hypothesis, PCPs who had been practicing longer were four times more likely to note concern for OSA, compared to those who were more recently trained. Finally, PCPs trained in internal medicine were ten times more likely to have concern for OSA, a finding which is not surprising given their training with adults, in whom OSA is more prevalent. 
Evidence-based guidelines recommend that snoring children with one or more additional signs receive a referral for further OSA evaluation. ${ }^{5}$ In the current study, when PCPs responded to automated prompts they had a concern for OSA in only $16 \%$ of snoring children. While we do not know how many children in the sample had at least one additional sign or symptom, the list of additional signs and symptoms in the published guidelines is broad and inclusive, including a total of 18 items. In our sample of snoring children for whom the PCP responded to the prompt, we know that $44 \%$ had at least one of the two risk factors measured in this study (ADHD symptoms and overweight). This strongly suggests there are many children who should be referred for further evaluation per guidelines, yet are not eliciting PCP concern for OSA.

A number of factors may contribute to the low proportion of concern for OSA. The average amount of time devoted to pediatric sleep medicine training in medical school and pediatric residency is 17 minutes and 4.4 hours, respectively. ${ }^{17,18}$ PCPs have performed similar to chance on tests of OSA knowledge and report low to moderate comfort in the diagnoses and management of OSA. ${ }^{23,24}$

A major strength of this study is the inclusion of a large sample of diverse families across five primary care clinic sites, as well as high caregiver responsiveness to the screening item. Additionally, participating PCPs were diverse in regards to type of training and years of practice. This study also has some limitations that warrant consideration. When PCPs did not suspect OSA, many questions remain unanswered as to the factors involved in d. Finally, findings may not fully generalize to practice patterns from other geographic areas and practices. These findings, however, are consistent with the literature showing problematic PCP identification and 
management of OSA across a variety of geographic areas within the United States and internationally. ${ }^{6}$

Altogether, this study highlights unwarranted practice variation in OSA suspicion in primary care. Given the huge burden placed on PCPs to address multiple health areas in a short time, strategies that support the provision of evidence-based care are critical. Automated decision support tools represent one potential for increasing access to care for children with OSA, though systems that include only screening for snoring are likely insufficient. An important next step is to build and evaluate a more detailed OSA module that will provide PCPs with additional support in evidence-based OSA identification and management. Supporting PCPs in evidencebased OSA identification may be an important first step towards the goal of access to quality care for children with OSA.

\section{Acknowledgements}

The authors would like to thank the dedicated providers and staff at the five participating Eskenazi Health clinic sites, as well as all of the participating children and families. We further wish to acknowledge the technical expertise and efforts of the individual members of the Child Health Informatics and Research Development Lab (CHIRDL) team, which provides programming and technical support for CHICA, and the Pediatric Research Network (PReSNet) at Indiana University School of Medicine for regulatory support. 


\section{References}

1. Bhattacharjee R, Kheirandish-Gozal L, Pillar G, Gozal D. Cardiovascular complications of obstructive sleep apnea syndrome: evidence from children. Prog Cardiovasc Dis. 2009;51(5):416-433. doi:S0033-0620(08)00023-6 [pii] 10.1016/j.pcad.2008.03.002.

2. Gozal D, Kheirandish-Gozal L. Neurocognitive and behavioral morbidity in children with sleep disorders. Curr Opin Pulm Med. 2007;13(6):505-509. doi:10.1097/MCP.0b013e3282ef6880.

3. Halbower AC, Mahone EM. Neuropsychological morbidity linked to childhood sleepdisordered breathing. Sleep Med Rev. 2006;10(2):97-107. doi:10.1016/j.smrv.2005.10.002.

4. Marcus CL, Brooks LJ, Draper KA, et al. Diagnosis and management of childhood obstructive sleep apnea syndrome. Pediatrics. 2012;130(3):e714-55. doi:10.1542/peds.2012-1672.

5. Marcus CL, Brooks LJ, Draper KA, et al. Diagnosis and management of childhood obstructive sleep apnea syndrome. Pediatrics. 2012;130(3):576-584. doi:10.1542/peds.2012-1671.

6. Honaker SM, Meltzer LJ. Sleep in pediatric primary care: A review of the literature. Sleep Med Rev. 2016;25:31-39. doi:10.1016/j.smrv.2015.01.004.

7. Erichsen D, Godoy C, Granse F, Axelsson J, Rubin D, Gozal D. Screening for sleep disorders in pediatric primary care: are we there yet? Clin Pediatr (Phila). 2012;51(12):1125-1129. doi:10.1177/0009922812464548.

8. Owens JA. The practice of pediatric sleep medicine: results of a community survey. Pediatrics. 2001;108(3):E51. http://www.ncbi.nlm.nih.gov/pubmed/11533369. 
9. Wennberg J. Tracking Medicine: A Researcher's Quest to Understand Health Care. Oxford University Press; 2010.

10. Carroll A, Anand V, Dugan T. Increased physician diagnosis of asthma with the child health improvement through computer automation decision support system. Pediatr Allergy,. 2012.

11. Carroll A, Bauer N, Dugan T. Use of a computerized decision aid for developmental surveillance and screening: a randomized clinical trial. JAMA. 2014.

12. Anand AV, Carroll AAAE, Bauer NSN, et al. Patient-tailored prioritization for a pediatric care decision support system through machine learning. AMIA Anпu Symp Proc. 2013;2008(3):amiajnl-2013-001865. doi:10.1542/peds.2013-0933.

13. Anand V, Carroll A, Biondich P, Dugan T. Pediatric decision support using adapted Arden Syntax. Artif Intell. 2015.

14. Anand V, Carroll AE, Downs SM. Automated primary care screening in pediatric waiting rooms. Pediatrics. 2012;129(5):e1275-e1281.

15. Bauer N, Carroll A, Saha C. Experience with decision support system and comfort with topic predict clinicians' responses to alerts and reminders. JAMIA. 2015.

16. Downs SM, Anand V, Dugan TM, Carroll AE. You can lead a horse to water: physicians' responses to clinical reminders. In: AMIA Annual Symposium Proceedings. Vol 2010. American Medical Informatics Association; 2010:167.

17. Mindell JA, Bartle A, Ahn Y, et al. Sleep education in pediatric residency programs: a cross-cultural look. BMC Res Notes. 2013;6:130. doi:10.1186/1756-0500-6-130.

18. Mindell JA, Bartle A, Wahab NA, et al. Sleep education in medical school curriculum: a glimpse across countries. Sleep Med. 2011;12(9):928-931. 
doi:10.1016/j.sleep.2011.07.001.

19. Mindell JA, Owens JA. Sleep problems in pediatric practice: clinical issues for the pediatric nurse practitioner. J Pediatr Heal Care. 2003;17(6):324-331. doi:S0891524503002153 [pii].

20. Wennberg J. Practice variation: implications for our health care system. Manag Care. 2004.

21. Lumeng JC, Chervin RD. Epidemiology of pediatric obstructive sleep apnea. Proc Am Thorac Soc. 2008;5(2):242-252. doi:10.1513/pats.200708-135MG.

22. Flores G. Language barriers to health care in the United States. N Engl J Med. 2006;355(3):229-231. doi:10.1056/NEJMp058316.

23. Tamay Z, Akcay A, Kilic G, Suleyman A, Ones U, Guler N. Are physicians aware of obstructive sleep apnea in children? Sleep Med. 2006;7(7):580-584. doi:10.1016/j.sleep.2006.04.004.

24. Uong EC, Jeffe DB, Gozal D, et al. Development of a measure of knowledge and attitudes about obstructive sleep apnea in children (OSAKA-KIDS). Arch Pediatr Adolesc Med. 2005;159(2):181-186. doi:159/2/181 [pii]10.1001/archpedi.159.2.181. 
Figure 1. Study Flow Diagram

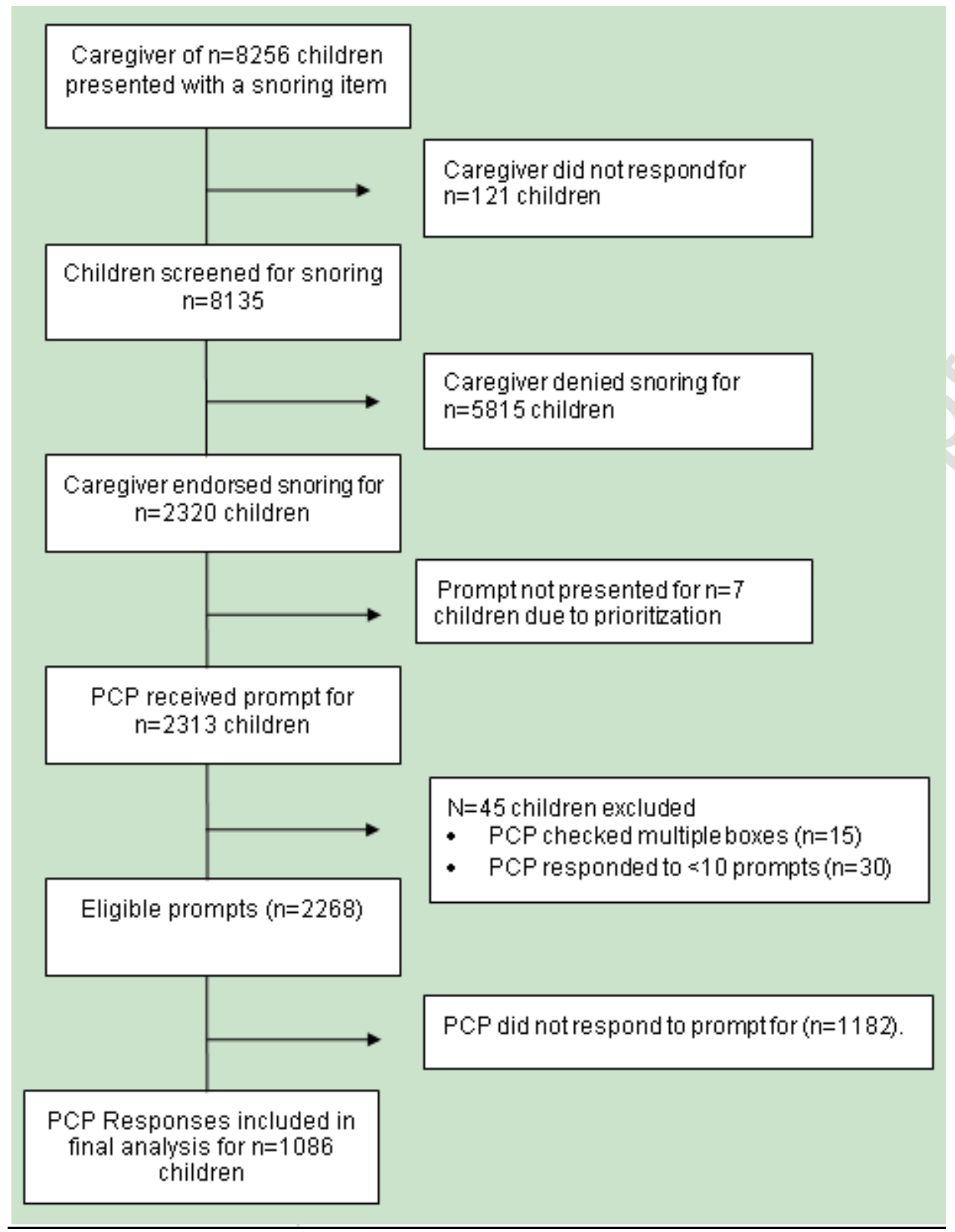


Figure 2: Variability in PCP Concern for OSA by Provider

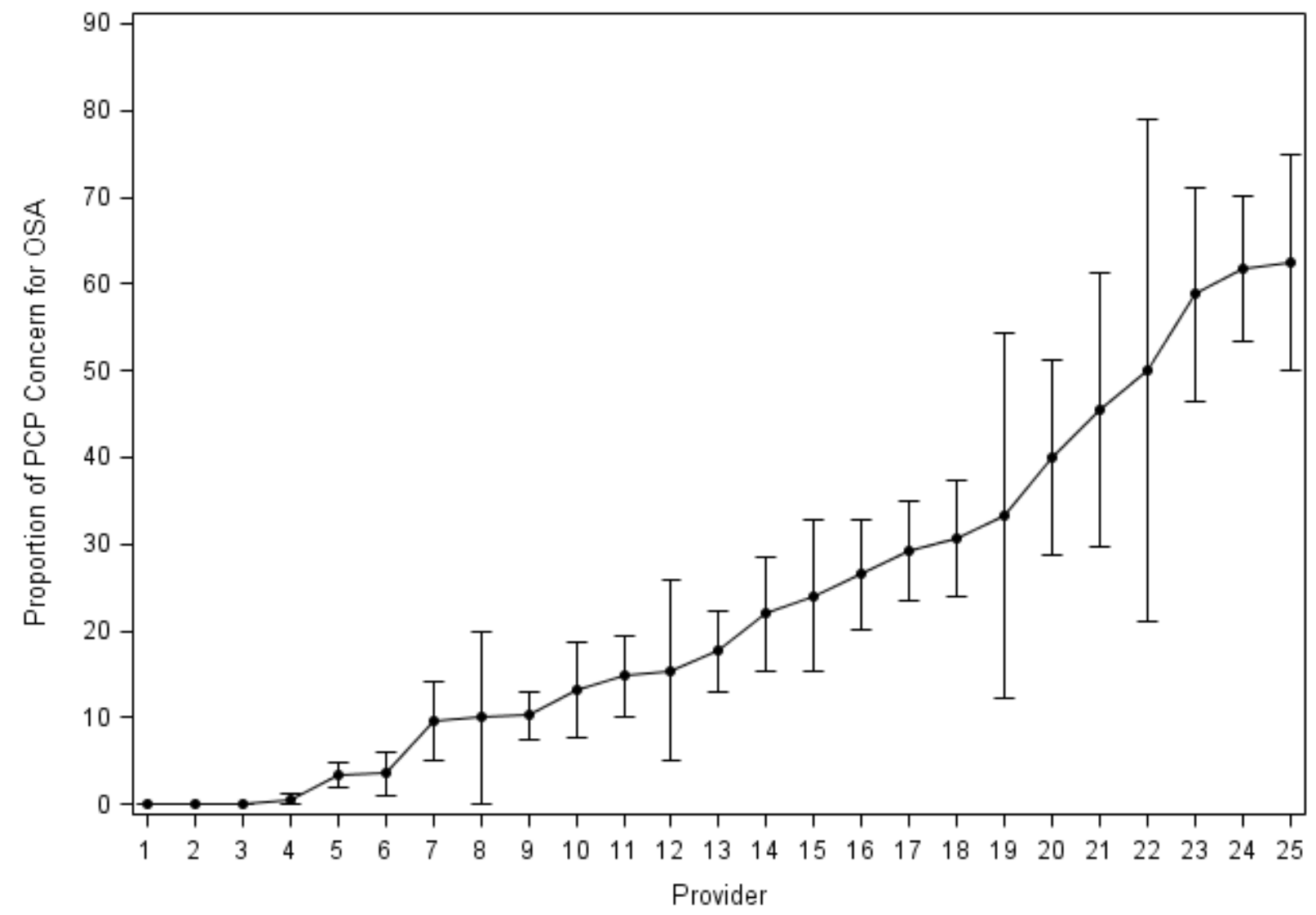


Table 1: Clinic, Child and Provider Characteristics

\begin{tabular}{|c|c|c|c|}
\hline Variables & Values & $\mathbf{N}$ & Percent \\
\hline \multirow{5}{*}{ Clinic } & 1 & 90 & 8.3 \\
\hline & 2 & 252 & 23.2 \\
\hline & 3 & 164 & 15.1 \\
\hline & 4 & 326 & 30.0 \\
\hline & 5 & 254 & 23.4 \\
\hline \multicolumn{4}{|l|}{$\begin{array}{l}\text { Child characteristics } \\
(n=1086)\end{array}$} \\
\hline Age (years) & Mean (SD) & $5.5(3.3)$ & \\
\hline \multirow{4}{*}{$\begin{array}{l}\text { Age group } \\
\text { (quartiles) }\end{array}$} & $1^{\text {st }}(1.0-2.5$ years $)$ & 278 & 25.6 \\
\hline & $2^{\text {nd }}(2.6-5.2$ years $)$ & 265 & 24.4 \\
\hline & $3^{\text {rd }}(5.3-8.2$ years $)$ & 269 & 24.8 \\
\hline & $4^{\text {th }}(8.3-11.9$ years $)$ & 274 & 25.23 \\
\hline \multirow{3}{*}{ Gender } & Male & 563 & 51.8 \\
\hline & Female & 521 & 48.0 \\
\hline & Missing & 2 & 0.2 \\
\hline \multirow{5}{*}{ Race } & Black & 475 & 43.7 \\
\hline & White & 57 & 5.3 \\
\hline & Hispanic & 302 & 27.8 \\
\hline & +2 & 224 & 20.6 \\
\hline & $+x$ & 28 & 2.6 \\
\hline \multirow{2}{*}{ Language } & English & 645 & 59.34 \\
\hline & Spanish & 441 & 40.6 \\
\hline \multirow{3}{*}{ Insurance } & Medicaid & 909 & 83.7 \\
\hline & Other & 66 & 6.1 \\
\hline & Missing & 111 & 10.2 \\
\hline \multirow{3}{*}{ BMI (2 category) } & Normal/ underweight & 469 & 43.2 \\
\hline & Overweight/ obese & 396 & 36.5 \\
\hline & Missing* & 221 & 20.4 \\
\hline \multirow{2}{*}{ ADHD symptoms } & No & 896 & 82.5 \\
\hline & Yes & 190 & 17.5 \\
\hline \multicolumn{4}{|l|}{$\begin{array}{l}\text { Provider characteristics } \\
(n=26)\end{array}$} \\
\hline \multirow{2}{*}{ Provider Gender } & Male & 18 & 69.2 \\
\hline & Female & 8 & 30.8 \\
\hline \multirow{4}{*}{ Provider Training } & Family & 4 & 15.4 \\
\hline & Med Peds & 3 & 11.5 \\
\hline & NP & 4 & 15.4 \\
\hline & Peds & 15 & 57.7 \\
\hline \multirow{2}{*}{ Years since training group } & $\geq$ median (14 years) & 13 & 50.0 \\
\hline & < median (14 years)) & 11 & 42.3 \\
\hline
\end{tabular}




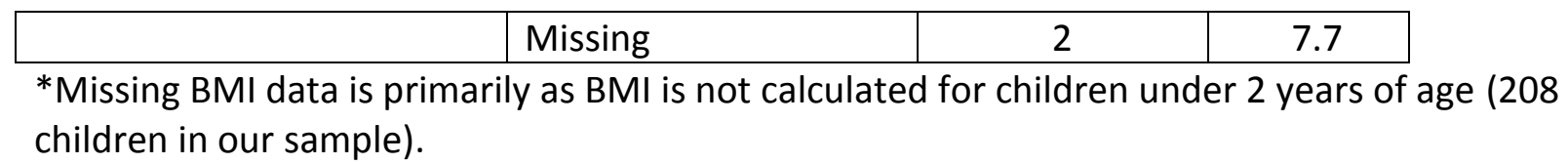

$\mathrm{BMI}=$ Body mass index; ADHD = Attention Deficit / Hyperactivity Disorder; Family = providers completing family medicine residency; Peds = providers completing pediatric residency; Meds Peds = providers completing a combined internal medicine and pediatric residency; $\mathrm{NP}=$ nurse practitioner. 
Table 2: Final Multivariate Model for the Outcome of PCP Suspicion for OSA

\begin{tabular}{|c|c|c|c|c|c|}
\hline Effects & Comparisons & OR & 95\% CI & P-value & $\begin{array}{l}\text { Overall } \\
\text { P-value }\end{array}$ \\
\hline \multirow{4}{*}{ Clinic } & 1 vs. 5 & 0.59 & {$[0.15,2.30]$} & 0.4497 & \multirow{4}{*}{0.0063} \\
\hline & 2 vs. 5 & 0.13 & {$[0.04,0.49]$} & 0.0025 & \\
\hline & 3 vs. 5 & 0.62 & {$[0.16,2.40]$} & 0.492 & \\
\hline & 4 vs. 5 & 1.25 & {$[0.42,3.72]$} & 0.6878 & \\
\hline \multirow{3}{*}{ Age } & $1.0-2.5$ vs. $8.3-11.9$ & 0.2 & {$[0.11,0.38]$} & $<.0001$ & \multirow{3}{*}{$<.0001$} \\
\hline & $2.6-5.2$ vs. $8.3-11.9$ & 0.5 & {$[0.30,0.83]$} & 0.0069 & \\
\hline & $5.3-8.2$ vs. $8.3-11.9$ & 1.01 & {$[0.63,1.60]$} & 0.9827 & \\
\hline $\begin{array}{l}\text { Primary } \\
\text { Language }\end{array}$ & Spanish vs. English & 0.53 & {$[0.34,0.82]$} & $\mathbf{0 . 0 0 5 0}$ & $\mathbf{0 . 0 0 5 0}$ \\
\hline \multirow{3}{*}{$\begin{array}{l}\text { Provider } \\
\text { Training }\end{array}$} & Family vs. Peds & 1.99 & {$[0.39,10.08]$} & 0.4062 & \multirow{3}{*}{0.0127} \\
\hline & Med Peds vs. Peds & 10.19 & {$[2.46,42.23]$} & 0.0014 & \\
\hline & NP vs. Peds & 2.61 & {$[0.53,12.76]$} & 0.2358 & \\
\hline Training Years & $\begin{array}{l}\geq \text { median (14) vs. }<\text { median } \\
\text { (14) }\end{array}$ & 4.26 & {$[1.41,12.92]$} & 0.0105 & 0.0105 \\
\hline
\end{tabular}

$\mathrm{PCP}=$ primary care provider; $\mathrm{OSA}=$ obstructive sleep apnea; $\mathrm{OR}=$ odds ratio; Family = providers completing family medicine residency; Peds = providers completing pediatric residency; Meds Peds = providers completing a combined internal medicine and pediatric residency; NP=nurse practitioner 